\title{
GROWTH INHIBITION OF TUBERCLE BACILLI BY ANALOGUES OF BIOTIN
}

\author{
HILDA POPE ${ }^{1}$ \\ Department of Bacteriology, Duke University, Durham, North Carolina \\ Received for publication July 17, 1951
}

Woods' report in 1940 on the mode of action of sulfanilamide initiated a new era in the study of chemotherapeutic agents. In the past decade hundreds of new drugs patterned after specific metabolites have been synthesized. Among these are a number of biotin analogues whose inhibitory activity for a variety of organisms has been described. In most instances activity of the compound has been limited to nonpathogenic organisms requiring an exogenous source of the vitamin, but biotin analogues have shown little activity against organisms that synthesize their own biotin.

The present paper reports the inhibitory activity of several biotin antagonists on a virulent strain of Mycobacterium tuberculosis. Our interest in these compounds lies not only in their potential chemotherapeutic value but also in their use in studies of biotin metabolism in the tubercle bacillus.

\section{MATERIALS AND METHODS}

A. In vitro studies. The virulent H37Rv strain of Mycobacterium tuberculosis was used in this study. Test cultures were made in Proskauer-Beck synthetic medium in 4-oz prescription bottles containing the analogue up to a final volume of $20 \mathrm{ml}$. One loopful of organisms from a young culture (2 to 4 weeks old) was used as the inoculum and was floated on the surface of the medium. The microscopic appearance and size of the pellicle were the criteria used in determining the effect of the compound upon the organism's growth during and at the end of a 6-week growth period. The analogues tested in this manner were 4-(imidazolidone-2) caproic acid, desthiobiotin, homobiotin, norbiotin, oxybiotin, and hexahydro-2-oxo-4-hydroxybutyl-1-furo-[3,4]imidazole.

A study utilizing the submerged type of growth for testing was undertaken for 4-(imidazolidone-2) caproic acid, homobiotin, and norbiotin. The substrate consisted of Dubos' sorbitan monooleate medium without albumen. This medium was also used in preparing serial dilutions of the added drugs, and tests were conducted in 18- $\mathrm{mm}$ matched test tubes containing a final volume of $10 \mathrm{ml}$. The inoculum consisted of $0.1 \mathrm{ml}$ of a 7-day culture of H37Rv organisms grown in the same basic medium. The cultures were shaken daily in order to maintain a homogeneous suspension throughout the growth period. At the end of a 10-day period of growth optical density readings were made on the cultures.

Concentrations of the drugs ranging from 1 to $100 \mathrm{mg}$ per cent were used.

1 This study was made possible through a grant from the Committee on Medical Research and Therapy of the American Trudeau Society, Medical Section of the National Tuberculosis Association. 
Duplicates were run at each concentration level, and four control cultures were included with each experiment. Additional studies were conducted with those drugs displaying an appreciable degree of inhibition, and the effect of biotin on this inhibition was tested. The minimal effective concentration of each drug bringing about complete inhibition was determined, and the antibacterial index was calculated. This is expressed as $C_{I} / C_{M}$ in which $C_{I}$ represents the minimum effective molar concentration of the analogue required to inhibit completely growth of the organism and $C_{M}$ which denotes the molar concentration of the vitamin sufficient to neutralize this inhibition.

Oxybiotin, desthiobiotin, pimelic acid, aspartic acid, and the other B-complex vitamins were tested for their ability to neutralize the inhibition produced by 4-(imidazolidone-2) caproic acid and homobiotin.

B. Testing of 4-(imidazolidone-2) caproic acid in the chick embryo. Because of the limited supply of the most active drug, 4-(imidazolidone-2) caproic acid, the intravenous injection of chick embryos (Lee et al., 1946) rather than the guinea pig method was employed. Since the H27Rv strain produced inconsistent results, the A-27 strain of Mycobacterium tuberculosis (14 days on the Proskauer-Beck medium) in doses of $10^{7}$ bacilli was used. Cell counts of a uniform suspension of organisms were made in a Petroff-Hauser counting chamber and the filtrate diluted so that the desired concentration was contained in $0.05 \mathrm{ml}$ of physiological saline solution.

4-(Imidazolidone-2) caproic acid was administered to infected embryos by dropping $0.3 \mathrm{ml}$ of a physiological saline solution containing $4.5 \mathrm{mg}$ of the drug on the exposed chorioallantoic membrane of 12-day old embryos at the time of infection and on the second and fourth days following infection.

On the twentieth day of incubation all of the embryos were harvested and the livers and spleens removed for examination. A gross comparison of the relative size and appearance of the spleens of the treated, untreated, and normal embryos was made. Sections of these two organs were stained by the Ziehl-Neelsen method and with hematoxylin-eosin.

Additional studies were also made in which the chorioallantoic membrane was infected by direct implantation of A-27 tubercle bacilli on the surface. The inoculum consisted of approximately $10^{7}$ bacilli suspended in $0.1 \mathrm{ml}$ of physiological saline. 4-(Imidazolidone-2) caproic acid was administered in the same manner and in the same concentration as for the intravenous type of infection after control studies had shown the drug to be nontoxic for the embryo. A comparison of the number and size of the lesions on the membranes of treated and untreated animals was made.

\section{RESULTS}

In vitro results. Of the six biotin analogues tested, 4-(imidazolidone-2) caproic acid showed the greatest inhibitory activity. $0.5 \mathrm{mg}$ per cent of the analogue resulted in complete inhibition of growth. This activity was neutralized when $0.005 \mathrm{mg}$ per cent of biotin was included in the test medium along with the analogue. The concentration of biotin was not critical because this amount of 
the vitamin was sufficient to neutralize concentrations of 4-(imidazolidone-2) caproic acid greater than the minimal effective concentration. There was no linear relationship between the concentrations of the analogue and the amount of biotin required to overcome the bacteriostatic effect of the analogue as indicated in table 1. The effectiveness of the drug was not decreased by testing in the sorbitan monooleate medium, and the minimal effective concentration of analogue and the amount of biotin required to neutralize its antibacterial activity remained the same. Aspartic acid, a mixture of several other amino acids and vitamins, serum, and pimelic acid were ineffective as reversing agents.

Desthiobiotin did not possess antibacterial activity but under certain conditions was able to substitute for biotin. When $0.05 \mathrm{mg}$ per cent of desthiobiotin

TABLE 1

Growth inhibition of the tubercle bacillus by 4-(imidazolidone-2) caproic acid and its reversal by biotin in sorbitan monooleate media

\begin{tabular}{|c|c|c|}
\hline 4-(RTDAZOLIDONE-2) CAPROIC ACD & BIOTIN & OPTICAL DENSTTY $(D=-$ LOG T $) \times 10$ \\
\hline mg per cent & mg per cent & \\
\hline 0.0 & 0.0 & 1.4 \\
\hline 0.05 & 0.0 & $1: 3$ \\
\hline 0.1 & 0.0 & 1.15 \\
\hline 0.1 & 0.0005 & 1.15 \\
\hline 0.1 & 0.001 & 1.2 \\
\hline 0.1 & 0.005 & 1.4 \\
\hline 0.5 & 0.0 & 0.0 \\
\hline 0.5 & 0.0001 & 0.1 \\
\hline 0.5 & 0.0005 & 0.2 \\
\hline 0.5 & 0.001 & 0.7 \\
\hline 0.5 & 0.005 & 1.35 \\
\hline 0.5 & 0.01 & 1.4 \\
\hline 5.0 & 0.0 & 0.0 \\
\hline 5.0 & 0.0001 & 0.0 \\
\hline 5.0 & 0.0005 & 0.15 \\
\hline 5.0 & 0.001 & 0.3 \\
\hline 5.0 & 0.005 & 1.4 \\
\hline 5.0 & 0.01 & 1.4 \\
\hline
\end{tabular}

was included in the test medium along with $0.5 \mathrm{mg}$ per cent of 4-(imidazolidone-2) caproic acid, the inhibitory effect of 4 -(imidazolidone-2) caproic acid was completely neutralized. $0.05 \mathrm{mg}$ per cent of oxybiotin also neutralized the activity of $0.5 \mathrm{mg}$ per cent of 4 -(imidazolidone-2) caproic acid.

On the surface of Proskauer-Beck medium, homobiotin, the higher homologue of biotin, completely prevented growth in concentrations of $10 \mathrm{mg}$ per cent; the activity being neutralized by $0.05 \mathrm{mg}$ per cent of biotin. In the sorbitan monooleate medium, however, $5 \mathrm{mg}$ per cent was sufficient to bring about the same degree of inhibition. The relation between the amount of analogue and the amount of biotin required for complete reversal of this activity in the sorbitan monooleate medium is shown in table 2 . In contrast to the findings with 4-(imi- 
dazolidone-2) caproic acid, here the relationship was linear, i.e., with increases in the amount of homobiotin above the minimal effective concentration, comparable increases in the amount of biotin are required for complete reversal. Five $\mathrm{mg}$ per cent of oxybiotin was also able to reverse the inhibitory effect of homobiotin.

TABLE 2

Growth inhibition of tubercle bacilli by homobiotin and its reversal by biotin in sorbitan monooleate media

\begin{tabular}{|c|c|c|}
\hline moMoBiotin & BIOTIN & OPTICAL DENSITY $(D=-$ LOG T $) \times 10$ \\
\hline mg per cent & mg per cent & \\
\hline 0 & 0.0 & 1.55 \\
\hline 1 & 0.0 & 1.38 \\
\hline 5 & 0.0 & 0.05 \\
\hline $\mathbf{5}$ & 0.005 & 0.05 \\
\hline 5 & 0.01 & 0.20 \\
\hline 5 & 0.05 & 1.53 \\
\hline 5 & 0.1 & 1.55 \\
\hline 10 & 0.0 & 0.05 \\
\hline 10 & 0.01 & 0.05 \\
\hline 10 & 0.05 & 1.13 \\
\hline 10 & 0.1 & 1.55 \\
\hline 50 & 0.0 & 0.05 \\
\hline 50 & 0.05 & 0.05 \\
\hline $\mathbf{5 0}$ & 0.1 & 0.05 \\
\hline 50 & 0.25 & 0.70 \\
\hline 50 & 0.5 & 1.54 \\
\hline
\end{tabular}

TABLE 3

Growth inhibition and its reversal in Hs7Rv tubercle bacilli

\begin{tabular}{|c|c|c|c|c|c|c|c|}
\hline \multirow{2}{*}{ ANALOGUE } & \multirow{2}{*}{$\begin{array}{c}\text { DNHIBI- } \\
\text { TORY } \\
\text { CONCEN- } \\
\text { TRATION } \\
\text { MG PEER } \\
\text { CENT }\end{array}$} & \multicolumn{2}{|c|}{ BIOTIN REVERSAL } & \multicolumn{2}{|c|}{$\begin{array}{l}\text { DESTHIOBIOTIN } \\
\text { REVERSAI }\end{array}$} & \multicolumn{2}{|c|}{$\begin{array}{l}\text { OXYBIOTIN REVER- } \\
\text { SAI }\end{array}$} \\
\hline & & $\underset{\text { per cent }}{\mathbf{m g}}$ & $\mathrm{C}_{\mathrm{I}} / \mathrm{C}_{\mathbf{x}}$ & $\underset{\text { per cent }}{\mathrm{mg}}$ & $\mathrm{C}_{\mathrm{T}} / \mathrm{C}_{\mathbf{n}}$ & $\underset{\text { per cent }}{\text { mg }}$ & $\mathrm{C}_{\mathbf{I}} / \mathrm{C}_{\mathbf{M}}$ \\
\hline 4-(Imidazolidone-2) caproic acid & 0.5 & 0.005 & 119.0 & 0.05 & 10.7 & 0.05 & 11.4 \\
\hline Homobiotin & 10.0 & 0.05 & 185.7 & None & 一 & 5.0 & 1.8 \\
\hline Norbiotin & 25.0 & 0.1 & 268.3 & None & - & 10.0 & 2.6 \\
\hline $\begin{array}{l}\text { Hexahydro-2-oxo-4-hydroxybutyl- } \\
\text { 1-furo-[3,4]imidazole }\end{array}$ & 10.0 & 0.05 & 257.1 & None & - & 5.0 & 2.5 \\
\hline
\end{tabular}

Norbiotin, the lower homologue of biotin, was found to have a minimal effective concentration of $25 \mathrm{mg}$ per cent with $0.1 \mathrm{mg}$ per cent of biotin required for reversal. A comparison of the antibacterial index of this analogue with that of homobiotin and 4-(imidazolidone-2) caproic acid shows it to be a less effective inhibitor (table 3).

Hexahydro-2-oxo-4-hydroxybutyl-1-furo-[3,4]imidazole also functioned as a biotin antagonist for the tubercle bacillus. A minimal effective concentration 
of $10 \mathrm{mg}$ per cent with neutralization by $0.05 \mathrm{mg}$ per cent of biotin was obtained.

With concentrations of desthiobiotin and oxybiotin up to $100 \mathrm{mg}$ per cent, no inhibition in growth was observed.

Results in the chick embryo. No difference could be detected either grossly or microscopically between the extent of liver and splenic involvement in the treated and untreated embryos. In the embryos infected via the chorioallantoic membrane, numerous lesions were present on the membrane in both treated and untreated groups. The only conclusion that could be made from these observations is that in the concentrations tested, 4-(imidazolidone-2) caproic acid had no retarding effect in the developing chick embryo upon the progression of infection with virulent tubercle bacilli.

\section{DISCUSSION}

The tubercle bacillus synthesizes a sufficient quantity of biotin to fulfill its nutritional needs (Pope and Smith, 1946). The data presented in this paper present further evidence that even in such organisms as the tubercle bacilli which have no exogenous requirement for essential growth factors, a nutritional deficiency may be produced. Since vitamins act as constituents of enzyme systems, the analogues could interfere with either the synthesis or the function of the active enzyme.

In the various compounds tested, the biotin molecule had been structurally modified in a variety of ways; 4-(imidazolidone-2) caproic acid was found to be the most effective inhibitor tested. This antagonist differs from biotin in that the sulfur ring has been opened and the sulfur removed from the molecule. The analogue resembles more closely desthiobiotin, differing from it only in the lack of the terminal methyl group.

For some organisms desthiobiotin has biotin-like activity, because of its in vivo conversion to biotin (Dittmer et al., 1944); for others desthiobiotin is inhibitory, presumably because it cannot be converted to biotin by these organisms (Melville et al., 1943). For the tubercle bacillus desthiobiotin functions as a metabolite and is able to neutralize the inhibitory activity of 4-(imidazolidone-2) caproic acid. From the results it is apparent that 4-(imidazolidone-2) caproic acid competes with desthiobiotin for an enzyme system which converts desthiobiotin to biotin, the function of which is essential for growth. The antibacterial index is a function of this inhibited enzyme system and is for this competitive inhibition about 10. The antibacterial index of an antimetabolite is calculated on the basis of its neutralization by a particular metabolite. Since 4-(imidazolidone2) caproic acid is more correctly termed an analogue of desthiobiotin rather than of biotin, this antibacterial index of 10 would be useful in characterizing the antibacterial properties of 4 -(imidazolidone-2) caproic acid against tubercle bacilli.

The effect of biotin on the toxicity of 4-(imidazolidone-2) caproic acid apparently corresponds to that of the product of the inhibited enzyme system. The inability to demonstrate a linear relationship between various concentrations of the drug and the minimal effective concentration of biotin required to overcome 
the bacteriostatic effect further emphasizes the role of biotin/ as the product of the inhibited enzyme system.

Homobiotin and norbiotin, the higher and lower homologue, respectively, of biotin, were also effective inhibitors. The site of action of these drugs, however, is at a different enzyme locus from that attacked by 4-(imidazolidone-2) caproic acid. Here the interference is with biotin itself, as evidenced by a competitive antagonism over a wide range of concentrations. Desthiobiotin, even in high concentrations, was unable to neutralize the inhibitory effect, whereas with oxybiotin neutralization of the inhibitory activity of homobiotin was complete. It may be concluded from the results obtained that the action of homobiotin probably lies in its prevention of the fixation of biotin to certain cellular constituents and its conversion to the enzymatically active form.

It has been postulated that the major quantitative requirement for biotin is in the synthesis of aspartic acid, while smaller quantities are required to catalyze the synthesis of oleic acid (Williams et al., 1947; Potter and Elvehjem, 1948). With the tubercle bacillus, however, the activity of 4-(imidazolidone-2) caproic acid and homobiotin was just as great when tests were made in the presence of aspartic acid and sorbitan monooleate. This finding indicates that at least for the tubercle bacillus, biotin is involved in some metabolic function other than the synthesis of aspartate and oleic acid.

\section{ACKNOWLEDGMENTS}

The author is greatly indebted to the following persons for supplying the analogues studied: Dr. J. H. Aeschlimann, Hoffman-La Roche, Inc., Nutley, New Jersey; Dr. Klaus Hofman, University of Pittsburgh, Pittsburgh, Pennsylvania.

\section{SUMMARY}

4-(Imidazolidone-2) caproic acid, homobiotin, norbiotin, and hexahydro-2oxo-4-hydroxybutyl-1-furo-[3,4]imidazole inhibited growth of virulent tubercle bacilli in low concentrations. Biotin neutralized the inhibitory activity of these compounds.

Desthiobiotin and oxybiotin exhibited no bacteriostatic action but functioned as a metabolite for the organisms. Desthiobiotin neutralized the inhibition produced by 4-(imidazolidone-2) caproic acid, and oxybiotin neutralized that produced by homobiotin, norbiotin, and 4-(imidazolidone-2) caproic acid.

From the data obtained it was concluded that 4-(imidazolidone-2) caproic acid inhibits growth of the tubercle bacillus by competing with an enzyme system which converts desthiobiotin to biotin. Homobiotin probably prevents the conversion of biotin to its enzymatically active form.

Aspartic acid and sorbitan monooleate had no effect on the bacteriostatic activity of these biotin analogues.

When tested in the chick embryo, 4-(imidazolidone-2) caproic acid was ineffective in checking the course of infection in embryos infected either intravenously or via the chorioallantoic membrane. 


\section{REFERENCES}

Dittmer, K., Melville, D. B., ANd dU Vigneadd, V. 1944 The possible synthesis of biotin from desthiobiotin by yeast and the anti-biotin effect of desthiobiotin for Lactobacillus casei. Science, 99, 203-205.

Lee, H. F., Stavitsky, A. B., and Lee, M. P. 1946 A chick embryo technic for intravenous and chemotherapeutic studies. Proc. Soc. Exptl. Biol. Med., 61, 143-149.

Melville, D. B., Ditmmer, K., Brown, G. B., and du Vigneaud, V. 1943 Desthiobiotin. Science, 98, 497-499.

Pope, H., and Smith, D. T. 1946 Synthesis of B-complex vitamins by tubercle bacilli when grown on synthetic media. Am. Rev. Tuberc., 54, 559-563.

Potter, R. L., AND Elvehjem, C. A. 1948 Biotin and the metabolism of Lactobacillus arabinosus. J. Biol. Chem., 172, 531-537.

Williams, W. L., Broquist, H. P., and Snell, E. E. 1947 Oleic acid and related compounds as growth factors for lactic acid bacteria. J. Biol. Chem., 170, 619-630.

Woods, D. D. 1940 The relation of $p$-aminobenzoic acid to the mechanism of the action of sulphanilamide. Brit. J. Exptl. Path., 21, 74-90. 